\title{
FINITELY PRESENTED MODULES OVER SEMIPERFECT RINGS
}

\author{
LOUIS H. ROWEN
}

\begin{abstract}
Results of Bjork and Sabbagh are extended and generalized to provide a Krull-Schmidt theory over a general class of semiperfect rings which includes left perfect rings, right perfect rings, and semiperfect PI-rings whose Jacobson radicals are nil.
\end{abstract}

The object of this paper is to lay elementary foundations to the study of f.g. (i.e. finitely generated) modules over rings which are almost Artinian, with the main goal being a theory following the lines of the Azumaya-Krull-Remak-SchmidtWedderburn theorem (commonly called Krull-Schmidt); in other words we wish to show that a given f.g. module is a finite direct sum of indecomposable submodules whose endomorphism rings are local. Previous efforts in this direction include [2, $\mathbf{3}, \mathbf{4}, \mathbf{6}]$, and in particular the results here extend some results of $[\mathbf{2}, \mathbf{4}, \mathbf{6}]$. The focus here will be on a "Fitting's lemma" approach applied to semiperfect rings, $\mathrm{cf}$. Theorem 8.

We recall the definition from $[\mathbf{1}]$, which will be used as a standard reference. $R$ is semiperfect if its Jacobson radical $J$ is idempotent-lifting and $R / J$ is semisimple Artinian; equivalently every f.g. module $M$ has a projective cover (an epic map $\pi: P \rightarrow M$, where $P$ is projective and $\operatorname{ker} \pi$ is a small submodule of $P$ ). Projective covers are unique up to isomorphism by [1, Lemma 17.17]. In what follows, module means "left module".

PROPOSITION 1. If $R$ is semiperfect, then every f.g. module $M$ is a finite direct sum of indecomposable submodules.

ProOF. Let $\pi: P \rightarrow M$ be a projective cover. Then $P$ has an indecomposable decomposition of some length (cf. [1, Theorem 27.12]) and we show by induction on $t$ that $M$ also has an indecomposable decomposition of length $\leq t$. Indeed this is tautological if $M$ is indecomposable, so assume $M=M_{1} \oplus M_{2}$. By [1, Lemma 17.17] there are projective covers $\pi_{i}: P_{i} \rightarrow M_{i}$, where $P_{i}$ are direct summands of $P$, and in fact $P_{1} \oplus P_{2} \approx P$ by $[\mathbf{1}$, Exercise 15.1], so we can proceed inductively on $M_{1}$ and $M_{2}$. Q.E.D.

REMARK 2. By [1, Theorem 27.6] an f.g. $R$-module $M$ is a direct sum of (indecomposable) modules having local endomorphism iff $\operatorname{End}_{R} M$ is semiperfect, so we ask: For which modules $M$ is $\operatorname{End}_{R} M$ semiperfect? (This is why it is natural to study semiperfect rings $R$.) In [4, Example 2.1] Bjork found an example of a cyclic module $M=R / L$ over a semiprimary $\operatorname{ring} R$ such that $E=\operatorname{End}_{R} M$ is not 1985.

Received by the editors January 4, 1984 and, in revised form, December 18, 1984 and May 17,

1980 Mathematics Subject Classification. Primary 16A51, 16A65; Secondary 16A50, 16 A64.

Key words and phrases. Semiperfect ring, indecomposable module, finitely presented module, Fitting's lemma, left $\pi$-regular, $\pi_{\infty}$-regular. 
semiperfect (because $E / \mathrm{Jac}(E)$ is a commutative ring which is not a field). Note his example also yields an onto map $M \rightarrow M$ which is not one-to-one. Our main results will therefore be about finitely presented modules $M$, i.e., $M=F / K$ where $F$ is an f.g. free module and $K$ is f.g., but we shall also say what we can for $M$ merely f.g.

PROPOSITION 3. Suppose $R$ is semiperfect and $J=\operatorname{Jac}(R)$ is nil. If $R a=R a^{2}$ for some $a$ in $R$, then $R a$ is a direct summand of $R$, i.e., $R a=R e$ for some idempotent $e$.

PROOF. Write $a=b a$ for some $b$ in $R a$. Then $b a=b^{2} a$, so $b^{2}-b \in R a \cap$ Ann $a$. Letting ${ }^{-}$be the image in $R / J$ we have $\overline{R a^{2}}=\overline{R a}$. Right multiplication by $\bar{a}$ gives a surjection $\psi: \overline{R a} \rightarrow \overline{R a}$ which is an isomorphism since $\bar{R}$ is semisimple Artinian. Then $\overline{b^{2}-b} \in \overline{R a} \cap \operatorname{Ann} \bar{a}=\operatorname{ker} \psi=0$, so $\bar{b}$ is idempotent in $\bar{R}$; hence $b^{2}-b$ is nilpotent. For some $k$ we have $0=\left(b-b^{2}\right)^{k}=b^{k}-p(b) b^{k+1}$ where $p(\lambda)$ is a polynomial in $\mathbf{Z}[\lambda]$, the sum of whose coefficients is 1 .

Let $e=(p(b) b)^{k}$ be an idempotent in $R$ whose image in $\bar{R}$ is $\bar{b}$. Then $e a=a$ since $b^{i} a=a$ for all $i$, and clearly $e \in R a$. Write $e=r a$. Of course, as $R$-modules, length $\overline{R e} \geq$ length $\overline{R e a}=$ length $\overline{R a}$; since $\overline{R e} \leq \overline{R a}$, we have $\overline{R e}=\overline{R a}$. Also length $\overline{R r} \geq$ length $\overline{R e}$. On the other hand,

$$
\text { (ere) } a=e r(e a)=e r a=e^{2}=e,
$$

so replacing $r$ by ere we have $r \in e R e$. In particular $\overline{R r} \leq \overline{R e}$ so $\overline{R r}=\overline{R e}$. Write $\bar{e}=\overline{r^{\prime} r}=\overline{r^{\prime} e r}=\overline{\left(e r^{\prime} e\right)(e r e)}$. Thus $\bar{r}$ is invertible in $\overline{e R e}$ implying $\overline{r+1-e}$ is invertible in $\bar{R}$. Thus $r+1-e$ is invertible in $R$ (since invertibility lifts up the Jacobson radical). But $(r+1-e) a=r a+a-e a=r a=e$, implying $R a=R e$, as desired. Q.E.D.

If we are to have a theory of modules satisfying a version of Fitting's lemma, then certainly $\operatorname{Jac}(R)$ must be nil when $R$ is local (taking $M=R$ ); in fact a somewhat stronger condition is needed, used in $[\mathbf{2}, \mathbf{5}]$ :

A ring $R$ is called left $\pi$-regular if it satisfies the DCC on chains of the form $R a>R a^{2}>R a^{3}>\cdots$. This condition is left-right symmetric by [5]. Note that if $R$ is left $\pi$-regular, then $\operatorname{Jac}(R)$ is nil (for if $a^{n} \in R a^{n+1}$, then $a^{n}=r a^{n+1}$ for some $r$, implying $(1-r a) a^{n}=0$, and thus $\left.a^{n}=0\right)$. On the other hand, [2, Proposition 2.3 ] shows that Fitting's lemma holds for $R$ (as an $R$-module) iff $R$ is left $\pi$-regular, so this class of rings is clearly of interest to us. Actually we are interested in a slightly stronger condition, in order to deal with arbitrary f.g. modules. Let us say $R$ is $\pi_{\infty}$-regular if $M_{n}(R)$ is left $\pi$-regular for each $n$. (Since this condition also is left-right symmetric we have dropped the word "left"; Dischinger [5] uses the terminology "completely $\pi$-regular".)

Any right perfect ring $R$ is $\pi_{\infty}$-regular since each matrix ring over $R$ is also right perfect and thus satisfies the descending chain condition on principal left ideals. Thus [5] implies any left perfect ring $R$ also is $\pi_{\infty}$-regular. There is an example of a semiperfect ring whose Jacobson radical is nil which is not $\pi_{\infty}$-regular, cf. [10], but such an example is rather hard to come by; a more thorough discussion is given in the appendix, which provides some positive results concerning when a given semiperfect ring is left $\pi$-regular. 
REMARK 4. Suppose $f: M \rightarrow M$ and $g: M \rightarrow N$ are maps of $R$-modules with $g f M=g M$. Then $M=f M+\operatorname{ker} g$. (This is standard: If $x \in M$, then $g x=g f y$ for some $y$, so $x-f y \in \operatorname{ker} g$.)

REMARK 5. If $f: P \rightarrow M$ is a projective cover and $P=P_{1} \oplus P_{2}$, then letting $f_{i}$ denote the restriction of $f$ to $P_{i}$ we have projective covers $f_{i}: P_{i} \rightarrow f P_{i}$ for $i=1,2$. (Indeed the $P_{i}$ are projective so it suffices to show $\operatorname{ker} f_{i}$ is small in $P_{i}$. If $N+\operatorname{ker} f_{1}=P_{1}$, then $N+P_{2}+\operatorname{ker} f=P$, implying $N+P_{2}=P$, so $N=P_{1}$.)

PROPOSITION 6. Suppose $R$ is a semiperfect ring with $M_{n}(\operatorname{Jac}(R))$ nil for all $n$ (e.g., this holds when $\operatorname{Jac}(R)$ is locally nilpotent). If $P$ is an f.g. projective $R$ module and $f: P \rightarrow P$ satisfies $f P=f^{2} P$, then $P=f P \oplus \operatorname{ker} f$. (In particular $f$ restricts to an isomorphism from $f P$ to itself.)

ProOF. $P=f P+\operatorname{ker} f$ by Remark 4 , so it suffices to prove $f P \cap \operatorname{ker} f=0$. Writing $P \oplus P^{\prime}=F$ f.g. free and extending $f$ to $F$ by putting $f x=x$ for all $x$ in $P^{\prime}$, we may thereby assume $P$ is free. But $f$ now acts as right multiplication by some matrix $a$ in $M_{n}(R)$, viewing the elements of $P$ as row vectors. Filling in with zeros underneath to view $P \subset M_{n}(R)$ we apply Proposition 3 to $M_{n}(R)$ to see $M_{n}(R) a$ is a direct summand of $M_{n}(R)$ as an $M_{n}(R)$-module. Hence $P a$ is a direct summand of $P$ as an $R$-module, implying $f P$ is a projective module, and $f: f P \rightarrow f P$ is onto and thus an isomorphism. (This is well known and has the following easy argument. As above we may assume $f P$ is free, so $f$ acts by right multiplication. This assertion is true for semisimple Artinian rings and thus for semilocal rings.) Thus $f P \cap \operatorname{ker} f=0$, as desired. Q.E.D.

PROPOSITION 7. Suppose $R$ is a semiperfect, $\pi_{\infty}$-regular ring. If $M$ is an f.g. $R$-module and $f: M \rightarrow M$ is a map, then $f^{t} M=f^{t+1} M$ for some $t$.

Proof. A standard trick enables us to assume $M$ is cyclic (for if $M$ is spanned by $n$ elements, i.e., $M=\sum_{i=1}^{n} R x_{i}$, then $M^{(n)}$ is a cyclic $M_{n}(R)$-module generated by $\left(x_{1}, \ldots, x_{n}\right)$, so we can replace $R$ by $M_{n}(R)$ and $M$ by $\left.M^{(n)}\right)$. But now writing $M=R x$ we have $f x=a x$ for some $a$ in $R$ and $a^{t} \in R a^{t+1}$ for some $t$ (by hypothesis), implying $f^{t} M \subseteq f^{t+1} M$, so $f^{t} M=f^{t+1} M$. Q.E.D.

THEOREM 8. Suppose $R$ is a semiperfect, $\pi_{\infty}$-regular ring, and $M$ is a finitely presented $R$-module.

(i) If $f: M \rightarrow M$ satisfies $f M=f^{2} M$, then $M=f M \oplus \operatorname{ker} f$.

(ii) "Fitting's lemma" : If $M$ is indecomposable, then every endomorphism of $M$ is either invertible or nilpotent, so in particular End $M$ is a local ring whose Jacobson radical is nil.

(iii) There is a decomposition $M=\bigoplus_{i=1}^{t} M_{i}$, unique up to permutation, such that each $M_{i}$ is indecomposable.

(iv) End $_{R} M$ is semiperfect and $\pi_{\infty}$-regular.

ProOF. (i) Remark 4 shows $M=f M+\operatorname{ker} f$, so we need only show $f M \cap \operatorname{ker} f=$ 0 ; a fortiori it suffices to show for some $t$ that $0=\operatorname{ker} f^{t} \cap f M=\operatorname{ker} f^{t} \cap f^{t} M$. As in [6, p. 77], take a projective cover $\pi: P \rightarrow M$ (by assumption $P$ and $\operatorname{ker} \pi$ are f.g.) and also there is $g: P \rightarrow P$ such that $\pi g=f \pi$, i.e., $g$ completes the diagram

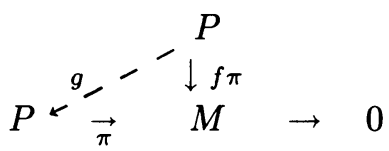


Clearly $g(\operatorname{ker} \pi) \subseteq \operatorname{ker} \pi$ (for if $\pi x=0$, then $\pi(g x)=f \pi x=0$ ), so Proposition 7 shows for large enough $t$ that $g^{t}(\operatorname{ker} \pi)=g^{t+1}(\operatorname{ker} \pi)$ and $g^{t} P=g^{t+1} P$. Proposition 6 implies $P=g^{t} P \oplus \operatorname{ker} g^{t}$, and $g^{t}$ restricts to an isomorphism from $g^{t} P$ to $g^{t} P$. Note $\pi g^{t}=f^{t} \pi$ by iteration.

Let $\pi^{\prime}$ be the restriction of $\pi$ to $g^{t} P$. By Remark 5 we have a projective cover $\pi^{\prime}: g^{t} P \rightarrow \pi^{\prime} g^{t} P=f^{t} \pi P=f^{t} M$. Moreover $g^{t}(\operatorname{ker} \pi) \leq g^{t} P \cap \operatorname{ker} \pi \leq \operatorname{ker} \pi^{\prime}$ implying

$$
g^{t}\left(\operatorname{ker} \pi^{\prime}\right) \leq g^{t}(\operatorname{ker} \pi)=g^{2 t}(\operatorname{ker} \pi) \leq g^{t}\left(\operatorname{ker} \pi^{\prime}\right),
$$

so equality holds at each step.

$g^{2 t}(\operatorname{ker} \pi)=g^{t}\left(\operatorname{ker} \pi^{\prime}\right)$, so $g^{t}(\operatorname{ker} \pi)=\operatorname{ker} \pi^{\prime}$. Thus $\operatorname{ker} \pi^{\prime}$ is an f.g. module, and $f^{t} M$ is finitely presented.

Replacing $M, P, \pi$ respectively by $f^{t} M, g^{t} P$, and $\pi^{\prime}$, we may assume $f$ is an onto $\operatorname{map}, \pi=\pi^{\prime}\left(\operatorname{so} g(\operatorname{ker} \pi)=\operatorname{ker} \pi^{\prime}\right)$ and $g$ is an isomorphism. But then $g$ restricts to a monomorphism from ker $\pi$ to itself, which is thus an isomorphism by [2, Theorem 1.1]. Hence $\operatorname{ker} f=0$, so $f$ is an isomorphism, as desired.

(ii) This is standard, by (i) and Proposition 7.

(iii) The decomposition exists by Proposition 1 ; each $M_{i}$ has a local endomorphism ring by (ii), so the Krull-Schmidt theorem (Azumaya's formulation) shows the decomposition is unique.

(iv) Let $E=\operatorname{End}_{R} M$. By (iii) $M=\bigoplus M_{i}$ so letting $e_{i}$ be the projection from $E$ to $M_{i}$, we see the $e_{i}$ are a complete set of orthogonal primitive idempotents, and $e_{i} E e_{i} \approx \operatorname{End}_{R} M_{i}$ is local by (ii), so $E$ is semiperfect by [1, Corollary 27.7]. $E$ is $\pi_{\infty}$-regular by [2, Proposition 2.3] applied to direct sums of copies of $M$. Q.E.D.

In order to apply this theorem we present a variant of (iv).

PROPOSITION 9. Suppose $R$ is a semiperfect ring whose Jacobson radical $J$ is nil. If $M$ is an f.g. $R$-module and $E=\operatorname{End}_{R} M$, we have $\operatorname{Jac}(E)^{k} M \subseteq J M$ for some $k$.

Proof. Let $E^{\prime}=\operatorname{End}_{R}(M / J M) . E^{\prime}$ is semisimple Artinian by Morita theory, since $M / J M$ is f.g. over the semisimple Artinian ring $R / J$. There is a ring homomorphism $E \rightarrow E^{\prime}$ given by $f \rightarrow \bar{f}$ where $\bar{f}(x+J M)=f x+J M$. The image $J^{\prime}$ of $\operatorname{Jac}(E)$ is a nil subring (without 1 ) which is thus nilpotent. Hence $\left(J^{\prime}\right)^{k}=0$ for some $k$, so $\operatorname{Jac}(E)^{k} M \subseteq J M$. Q.E.D.

Corollary 10. Using notation as in Proposition 9, suppose $M$ is spanned by $n$ elements.

(i) If $J$ is locally nilpotent, then $\mathrm{Jac}(E)$ is locally nilpotent.

(ii) If $M_{n}(J)$ is left T-nilpotent, then $\operatorname{Jac}(E)$ is right T-nilpotent.

(iii) If $M_{n}(J)$ is right $T$-nilpotent, then $\operatorname{Jac}(E)$ is left $T$-nilpotent.

(iv) If $J$ is nilpotent, then $\operatorname{Jac}(E)$ is nilpotent.

PROOF. (i) Let $S$ be a finite $\operatorname{subset}$ of $\operatorname{Jac}(E)$, and write $M=\sum_{i=1}^{n} R x_{i}$. For $k$ as in the proposition we have $\left\{s x_{i}: s \in S^{k}, 1 \leq i \leq n\right\} \subseteq \sum_{i=1}^{n} J_{0} x_{i}$ for some finite subset $J_{0}$ of $J$, and thus $J_{0}^{q}=0$ for some $q$; hence $S^{k q} x_{i}=0$ for all $i$, so $S^{k q}=0$.

(ii), (iii) Passing to $M_{n}(R)$ and $M^{(n)}$ instead of $R$ and $M$ we have the same endomorphism ring $E$, so we may assume $M$ is cyclic (cf. proof of Proposition $7)$, i.e., $M=R x$. Let $f_{1}, f_{2}, \ldots$ be any sequence of elements of $\operatorname{Jac}(E)$. Then 
$\left(f_{1} \cdots f_{k}\right) x=a_{1} x$ for some $a_{1}$ in $J$, and in general $f_{k t+1} \cdots f_{k(t+1)} x=a_{t+1} x$ for $a_{t+1}$ in $J$, yielding

$$
f_{1} \cdots f_{k(t+1)} x=f_{1} \cdots f_{k t} a_{t+1} x=a_{t+1} f_{1} \cdots f_{k t} x=\cdots=a_{t+1} \cdots a_{1} x .
$$

If $J$ is left (resp. right) $T$-nilpotent we thereby $\operatorname{see} \operatorname{Jac}(E)$ is right (resp. left) $T$ nilpotent, as desired.

(iv) As in (ii) and (iii), noting that $J$ is nilpotent implies $M_{n}(J)$ is nilpotent.

COROllary 11. (Compare with Bjork [3, Theorems 4.1 and 4.2].) Suppose $M$ is a finitely presented $R$-module, and $E=\operatorname{End}_{R} M$. If $R$ is left perfect, then $E$ is right perfect; if $R$ is right perfect, then $E$ is left perfect; if $R$ is semiprimary, then $E$ is semiprimary.

ProOF. Combine Theorem 8 and Corollary 10, since these rings all are semiperfect and $\pi_{\infty}$-regular. Q.E.D.

Discussion of results. In this paper we have gone the route of Fitting's lemma, which holds by [2, Proposition 2.3] iff $\operatorname{End}_{R} M$ is left $\pi$-regular. Taking $M=R^{(n)}$ for each $n$, we see a necessary condition for Fitting's lemma to hold is for $R$ to be $\pi_{\infty}$-regular. On the other hand if we want a finitely presented module $M$ to be the direct sum of modules having local endomorphism rings, then in particular taking $M=R$ we see $R$ must be semiperfect (cf. [1, Corollary 27.7]). Thus the hypotheses of Theorem 8 are necessary for us to develop a Krull-Schmidt theory via Fitting's lemma and local endomorphism rings, and in this sense Theorem 8 is as strong as possible. However we have bypassed the question of classifying semiperfect $\pi_{\infty}$ regular rings in more intrinsic terms, such as the Jacobson radical. We shall address this question in the appendix and in [10].

On the other hand there are instances where a Krull-Schmidt theory can be obtained without Fitting's lemma. (For example if $P$ is an f.g. projective module over a semiperfect ring $R$, then $\operatorname{End}_{R} P$ is semiperfect by [1, Corollary 27.8].) In [9] we shall take up the question of what conditions on $R$ guarantee this for arbitrary finitely presented modules $P$.

Note that Proposition 9 also implies that if $M$ is spanned by $n$ elements and $R$ is semiperfect with $M_{n}(\operatorname{Jac}(R))$ nil, then $\operatorname{Jac}\left(\operatorname{End}_{R} M\right)$ is nil. (Proof: Pass to the cyclic case as usual, and writing $M=R x$ and $f^{k} x=a x$ for $f$ in $\operatorname{Jac}\left(\operatorname{End}_{R} M\right)$ and $a$ in $J$, note $a^{m}=0$ for some $m$, implying $f^{k m}=0$.)

Corollary 10 also shows in general that every nil subring of $\operatorname{End}_{R} M$ is nilpotent, whenever $M$ is an f.g. module over a semiprimary ring $R$.

Another kind of ring arising in these considerations is a ring $R$ which satisfies DCC on chains of principal left ideals of the form $R s_{1}>R s_{2} s_{1}>R s_{3} s_{2} s_{1}>\cdots$ whenever all $s_{i}$ are from a finite set $S$. It is easy to see that $\operatorname{Jac}(R)$ is then locally nilpotent. (Proof: Suppose $S$ is a finite subset of $\operatorname{Jac}(R)$ which is not nilpotent. Then there is $s_{1}$ in $S$ such that $S^{k} s_{1} \neq 0$ for all $k$, for otherwise if $S^{k(s)} s=0$, then $\max (k(s))+1$ would be a bound for the index of nilpotence of $S$. Continuing in this way one finds $s_{2}$ with $S^{k} s_{2} s_{1} \neq 0$ for all $k$, and so on, and clearly $R s_{1}>R s_{2} s_{1}>R s_{3} s_{2} s_{1}>\cdots$.) This seems to be a natural class of rings generalizing left perfect rings, so it would be nice to characterize them in terms of the Jacobson radical. 
Appendix: Examples of semiperfect left $\pi$-regular rings. The results of this paper apply to the class of semiperfect $\pi_{\infty}$-regular rings. This leads one to search for classes of examples, particularly ones which are not perfect. As we observed earlier, any such ring $R$ has $\operatorname{Jac}(R)$ nil. On the other hand if $R$ is semilocal and $\operatorname{Jac}(R)$ is nil, then $R$ is semiperfect (since nil ideals are idempotent-lifting), so we would like to be able to conclude that $R$ is $\pi_{\infty}$-regular. Unfortunately this need not be true. In this appendix we show a semilocal ring $R$ is $\pi_{\infty}$-regular when $\operatorname{Jac}(R)$ is the lower nilradical of $R$, in particular when $R$ is a PI-ring (i.e., a ring satisfying a polynomial identity).

Our method of approach is to see what conclusions can be drawn from the existence of a ring $R$ such that $J=\operatorname{Jac}(R)$ is nil and $R / J$ is semisimple Artinian, but $R$ is not left $\pi$-regular; we shall call such $R$ a counterexample.

LEMMA 12. If $R$ is a counterexample, then some prime homomorphic image of $R$ is a counterexample.

PROOF. We rely on an elegant result of $[\mathbf{7}$, Theorem 2.1$]$, in which it is shown some prime homomorphic $R / P$ image of $R$ is not left $\pi$-regular. Let $J=\operatorname{Jac}(R)=$ $\bigcap_{i=1}^{n} M_{i}$ for maximal ideals $M_{i}$ of $R$. (By hypothesis $J$ is nil and $R / J$ is semisimple Artinian, so $n$ is finite). Note that $M_{1}, \ldots, M_{n}$ are the only prime ideals of $R$ containing $J$. Reordering the $M_{i}$ we may assume $P \subset M_{i}$ for $1 \leq i \leq t$, suitable $t$.

Let $\operatorname{Nil}(R / P)=N / P$, for suitable $N \triangleleft R$. For any $a \notin N$ there is some ideal $P_{a} \supset P$ of $R$ maximal with respect to missing all powers of $a$. It is standard that $R / P_{a}$ is prime with nilradical 0 , so $J \subset P_{a}$, implying $P_{a}=M_{i}$ for suitable $1 \leq i \leq t$. Thus $\bigcap_{i=1}^{t} M_{i} \subseteq N$, and clearly $N \subseteq M_{i}$ for each $1 \leq i \leq t$, so $(R / P) /(N / P) \approx$ $R / \bigcap_{i=1}^{t} M_{i} \approx \prod_{i=1}^{t} R / M_{i}$ is semisimple Artinian, as desired. Q.E.D.

(Lemma 12 is very closely related to [5, Proposition 2].)

PROPOSITION 13. Suppose $R$ is semilocal and $\operatorname{Jac}(R)$ equals the lower nilradical $L(R)$ of $R$. Then $R$ is $\pi_{\infty}$-regular.

PROOF. First note that $R / L(R)$ is semisimple Artinian and thus left $\pi$-regular. Thus each prime image of $R$ is left $\pi$-regular, so $R$ is left $\pi$-regular by Lemma 12 . Furthermore the hypotheses pass to $M_{n}(R)$ for each $n ; \operatorname{Jac}\left(M_{n}(R)\right)$ is the lower nilradical of $M_{n}(R)$ (seen by viewing the lower nilradical as the intersection of all prime ideals), and $M_{n}(R)$ is semilocal, implying $M_{n}(R)$ is left $\pi$-regular. Hence $R$ is $\pi_{\infty}$-regular. Q.E.D.

COROLlaRY 14. If $R$ is a semilocal PI-ring and $\operatorname{Jac}(R)$ is nil, then $R$ is $\pi_{\infty}$ regular.

ProOF. By [8, Theorem 1.6.36] any nil ideal is in the lower nilradical. Q.E.D.

As stated earlier, there $i s$ a counterexample, which also provides a left $\pi$-regular ring which is not $\pi_{\infty}$-regular, thereby answering a long-standing question in the theory of $\pi$-regular rings. Since the construction is rather intricate, it will appear separately in $[\mathbf{1 0}]$. 


\section{REFERENCES}

1. F. Anderson and K. Fuller, Rings and categories of modules, Graduate Texts in Math., no. 13, Springer-Verlag, Berlin, New York and Heidelberg, 1973.

2. E. P. Armendariz, J. W. Fisher and R. L. Snider, On injective and surjective endomorphisms of finitely generated modules, Comm. Algebra 6 (1978), 659-672.

3. J. E. Bjork, Conditions which imply that subrings of semiprimary rings are semiprimary, J. Algebra 19 (1971), 384-395.

4. _ Conditions which imply that subrings of artinian rings are artinian, J. Reine Angew. Math. 247 (1971), 123-138.

5. F. Dischinger, Sur les anneaux fortement $\pi$-réguliers, C.R. Acad. Sci. Paris Ser. A-B 283 (1976), A571-A573.

6. G. Sabbagh, Endomorphism rings of finitely presented modules, Proc. Amer. Math. Soc. 30 (1971), 75-78.

7. J. W. Fisher and R. L. Snider On the von Neumann regularity of rings with regular prime factor rings, Pacific J. Math. 54 (1974), 135-144.

8. L. H. Rowen, Polynomial identities in ring theory, Pure and Applied Math., vol. 84, Academic Press, New York, 1980.

9. __ Finitely presented modules over semiperfect Noetherian rings, J. Algebra (to appear).

10. __ An example of a left $\pi$-regular ring, J. Algebra (to appear).

Faculty of Natural SCiences and Mathematics, Department of MathematICS, BAR-ILAN UNIVERSITY, RAMAT GAN, ISRAEL 